ARTICLE

\title{
Emergence of coherence in the charge-density wave state of $2 \mathrm{H}-\mathrm{NbSe}_{2}$
}

U. Chatterjee ${ }^{1,2}$, J. Zhao 2,3, M. lavarone4, R. Di Capua ${ }^{4}$, J.P. Castellan¹,5, G. Karapetrov6 , C.D. Malliakas ${ }^{1,7}$, M.G. Kanatzidis ${ }^{1,7}$, H. Claus ${ }^{1}$, J.P.C. Ruff ${ }^{8,9}$, F. Weber1,5, J. van Wezel1,10, J.C. Campuzano ${ }^{1,3}$, R. Osborn', M. Randeria ${ }^{11}$, N. Trivedi ${ }^{11}$, M.R. Norman ${ }^{1} \&$ S. Rosenkranz ${ }^{1}$

A charge-density wave (CDW) state has a broken symmetry described by a complex order parameter with an amplitude and a phase. The conventional view, based on clean, weak-coupling systems, is that a finite amplitude and long-range phase coherence set in simultaneously at the CDW transition temperature $T_{\mathrm{cdw}}$. Here we investigate, using photoemission, X-ray scattering and scanning tunnelling microscopy, the canonical CDW compound $2 \mathrm{H}-\mathrm{NbSe}_{2}$ intercalated with $\mathrm{Mn}$ and $\mathrm{Co}$, and show that the conventional view is untenable. We find that, either at high temperature or at large intercalation, CDW order becomes short-ranged with a well-defined amplitude, which has impacts on the electronic dispersion, giving rise to an energy gap. The phase transition at $T_{\text {cdw }}$ marks the onset of long-range order with global phase coherence, leading to sharp electronic excitations. Our observations emphasize the importance of phase fluctuations in strongly coupled CDW systems and provide insights into the significance of phase incoherence in 'pseudogap' states.

\footnotetext{
${ }^{1}$ Materials Science Division, Argonne National Laboratory, Argonne, Illinois 60439, USA. ${ }^{2}$ Department of Physics, University of Virginia, Charlottesville, Virginia 22904, USA. ${ }^{3}$ Department of Physics, University of Illinois at Chicago, Chicago, Illinois 60607, USA. ${ }^{4}$ Department of Physics, Temple University, Philadelphia, Pennsylvania 19122, USA. ${ }^{5}$ Institute of Solid State Physics, Karlsruhe Institute of Technology, PO Box 3640, D-76021 Karlsruhe, Germany. ${ }^{6}$ Department of Physics, Drexel University, Philadelphia, Pennsylvania 19104, USA. ${ }^{7}$ Department of Chemistry, Northwestern University, Evanston, Illinois 60208, USA. ${ }^{8}$ Advanced Photon Source, Argonne National Laboratory, Argonne, Illinois 60439, USA. ${ }^{9}$ CHESS, Cornell University, Ithaca, New York 14853 , USA. ${ }^{10}$ Institute for Theoretical Physics, University of Amsterdam, Tyndall Avenue, 1090 GL Amsterdam, The Netherlands. ${ }^{11}$ Department of Physics, Ohio State University, Columbus, Ohio 43210, USA. Correspondence and requests for materials should be addressed to U.C. (email: uc5j@virginia.edu) or to S.R. (email: srosenkranz@anl.gov).
} 
T he formation of charge-density waves (CDWs) and superconductivity are archetypical examples of symmetry breaking in materials, which are characterized by a complex order parameter. In clean weak-coupling systems, the formation of the amplitude and the establishment of macroscopic phase coherence are known to occur simultaneously at the transition temperature ${ }^{1,2}$, but the situation may be dramatically different at strong coupling or in the presence of disorder ${ }^{3-9}$. Such systems generally exhibit short correlation lengths and a transition temperature that is greatly suppressed from the expected mean-field value. This opens the possibility for a gap in the electronic spectra to persist in the absence of long-range order over a large temperature range and the opportunity to study this so-called pseudogap behaviour, which has been observed in a wide range of systems from high-temperature superconductors $^{10-12}$ to disordered superconducting thin films ${ }^{13}$ and cold atoms ${ }^{14}$, in a simple system.

In the CDW state below the transition temperature $T_{\mathrm{cdw}}$, a modulation appears in the density of conduction electrons that is accompanied by a periodic lattice distortion ${ }^{1}$. Although this lattice distortion leads to an increase in the elastic energy, there is a net gain in the free energy of the system due to a reduction in the energy of the electrons via the opening of an energy gap $\Delta$. The CDW state is characterized by the order parameter $\delta \rho(\mathbf{r})=\rho(\mathbf{r})-\rho_{\text {av }}(\mathbf{r})=\rho_{0} \cos [\mathbf{q} \cdot \mathbf{r}+\phi(\mathbf{r})]$, where $\mathbf{q}$ is the CDW wave vector, $\rho_{\text {av }}$ the average charge density, $\rho_{0}$ the amplitude of the CDW order parameter, which is proportional to the energy gap $\Delta$, and $\phi$ the phase of the CDW, that is, the location of the charge modulation with respect to the underlying lattice. It is straightforward to realize that there are two ways to destroy the order parameter-(i) by reducing the amplitude $\rho_{0}$ through excitations across the energy gap and/or (ii) by randomization of the phase $\phi$ either through thermal or quantum fluctuations. Similar to the case of Bardeen-Cooper-Schrieffer (BCS) transitions in clean superconductors, the expectation value, of $\delta \rho$ in weakly coupled CDW systems vanishes via (i), as, in general, (ii) is not relevant due to the large magnitude of the phase stiffness energy ${ }^{1,3}$. However, strongly coupled CDW systems can be susceptible to strong phase fluctuations due to their shorter coherence lengths $s^{1,4-7}$.

To explore this systematically as a function of disorder in a material with a simple electronic and crystal structure, we investigate pristine and intercalated (with $\mathrm{Co}$ and $\mathrm{Mn}$ ions) $2 \mathrm{H}$ - $\mathrm{NbSe}_{2}$ samples, where CDW ordering involves strong electron-phonon coupling ${ }^{15,16}$. In our investigations, we employ a combination of experimental probes. Scanning tunnelling microscopy (STM) and X-ray diffraction (XRD) are used to measure the real and momentum space structure of the CDW order, respectively. Angle-photoemission spectroscopy (ARPES) is employed to investigate the presence of an energy gap in the electronic spectra, which is proportional to the amplitude of the order parameter, and to infer the existence or
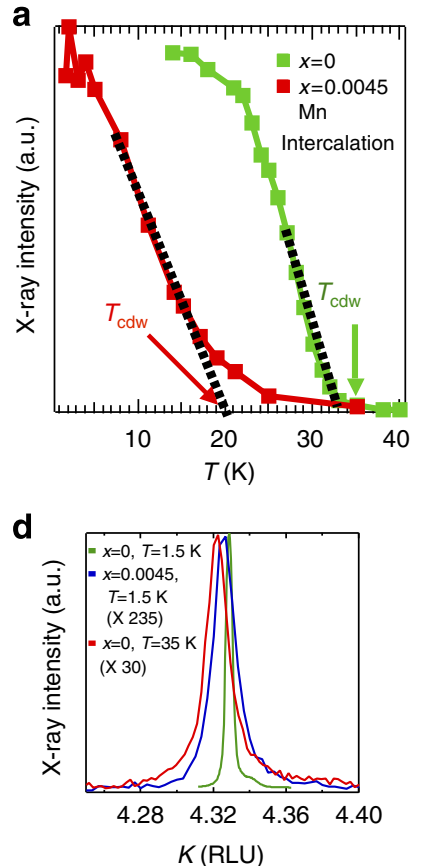

b

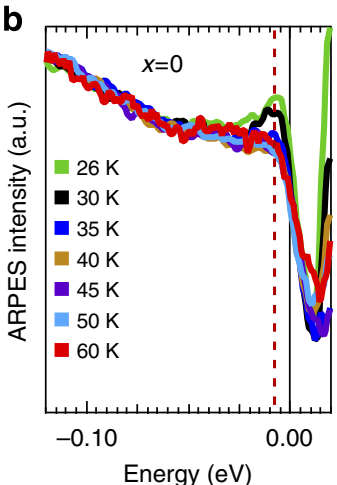

e

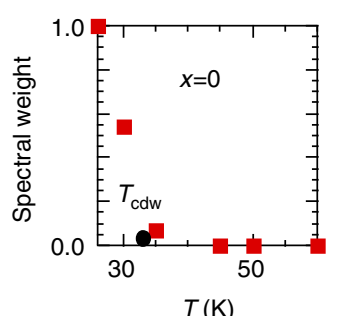

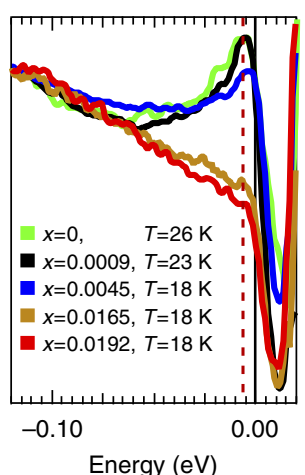

f

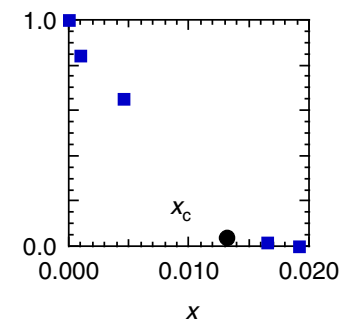

Figure 1 | CDW phase transition for pure and intercalated $\mathbf{2 H}-\mathbf{N b S e}_{\mathbf{2}}$ samples. (a) Intensity of the CDW superstructure peak as a function of $T$ for an $x=0$ (green) and $x=0.0045$ ( $M n$-intercalated, red). Dotted lines are guides to the eye, indicating the growth of the CDW order parameter. Arrows mark the estimated $T_{\mathrm{cdw}}$ which is the interpolation of the linear part to zero. (b) FF divided EDCs for various temperatures at the momentum location shown by the red dot in Fig. 2d. (c) FF divided EDCs for different $x$ at the lowest measured temperatures. The sample with $x=0.0165$ is intercalated with $\mathrm{Co}$, the others are intercalated with $\mathrm{Mn}$. The corresponding momentum location is shown by the pink dot in Fig. $2 \mathrm{~d}$. The red dashed line in $\mathbf{b}$ and $\mathbf{c}$ shows the energy location of the peak (for $T<T_{\mathrm{cdw}}$ and $x<x_{\mathrm{c}}$ ) and kink (for $T>T_{\mathrm{cdw}}$ or $x>x_{\mathrm{c}}$ ) in the EDCs, while the black line is the chemical potential. (d) Intensity profile of CDW superstructure peaks along $(0, K, 0)$ for $x=0,0.0045$ (intercalated with $\mathrm{Mn}$ ) at $1.5 \mathrm{~K}$ and for $x=0$ at $T=35 \mathrm{~K}$. The intensities have been normalized to that of the $(0,4,4)$ Bragg peak and have subsequently been multiplied by the factors displayed in the legend to make them all visible on the same scale. (e,f) Normalized spectral weight associated with the coherence peak, following the procedure as described below, as a function of $T$ for $x=0$ and as a function of $x$ at the lowest measured temperature. The black dots correspond to $T_{\mathrm{cdw}}$ for $x=0$ and $x_{\mathrm{c}} \sim 0.013$, respectively. For $x=0$, the coherent spectral weight was obtained as the remaining integrated spectral intensity after subtracting the highest measured temperature $(60 \mathrm{~K})$ as background and dividing by the value obtained at the lowest measured temperature. We adopt the same procedure, as for the normalized spectral weight as a function of $T$, for its $x$ dependence by considering the spectrum at the highest value of $x$ as the background and dividing the coherent spectral weight at the lowest measured temperature for each $x$ by the one for $x=0$. 
disappearance of phase coherence of the order parameter through the presence or absence of sharp coherence peaks in the ARPES spectra. Furthermore, $T_{\mathrm{cdw}}$ is determined by tracking the onset of the CDW order parameter in XRD and the CDW-induced anomaly in transport measurements. Our main results from this extensive set of measurements are as follows. First, the temperature $T_{\mathrm{cdw}}$, above which $\mathrm{CDW}$ phase coherence is destroyed, is suppressed with intercalation $x$ and vanishes at a quantum phase transition $(\mathrm{QPT})$ at $x_{\mathrm{c}}$. Second, the CDW state below $T_{\mathrm{cdw}}(x)$ and $x<x_{\mathrm{c}}$ has an energy gap and sharp electronic excitations. Finally, the CDW energy gap survives above $T_{\mathrm{cdw}}(x)$ and $x>x_{c}$ in the state, which has only short-range CDW correlations, but the electronic excitations are no longer welldefined.

\section{Results}

XRD and transport. Previous investigations of the CDW transition in $2 \mathrm{H}-\mathrm{NbSe}_{2}$ using neutron and XRD have shown that a superstructure with incommensurate wave vector $q=(1-\delta) a^{\star} / 3$, where $a^{*}=4 \pi / \sqrt{3} a$, lattice parameter $a=3.44 \AA$ and $\delta \sim 0.02$, appears below $T_{\text {cdw }}=33 \mathrm{~K}$ (ref. 17). However, the origin of the $\mathrm{CDW}$ and the exact nature of the $\mathrm{Nb}$ atom motion involved were only recently established using inelastic X-ray scattering ${ }^{16}$ and superspace crystallography ${ }^{18}$, respectively. To explore the evolution of the CDW transition with increasing disorder, we use synchrotron XRD and transport measurements on a number of intercalated samples (for details, see Methods section and Supplementary Fig. 1). Figure 1a shows the intensity of the incommensurate superstructure peaks, which is proportional to the square of the CDW order parameter. For the undoped compound, the superstructure intensity increases continuously from the temperature-independent background present above $T_{\mathrm{cdw}}$, as expected for a second-order phase transition. Moreover, the width of this superstructure peak, which is a measure of the inverse of the CDW correlation length, becomes resolution limited below $T_{\mathrm{cdw}}$, implying the presence of long-range order (Fig. 1d). For intercalated samples, the XRD superstructure peak exhibits a reasonably sharp onset of intensity (Fig. 1a) as a
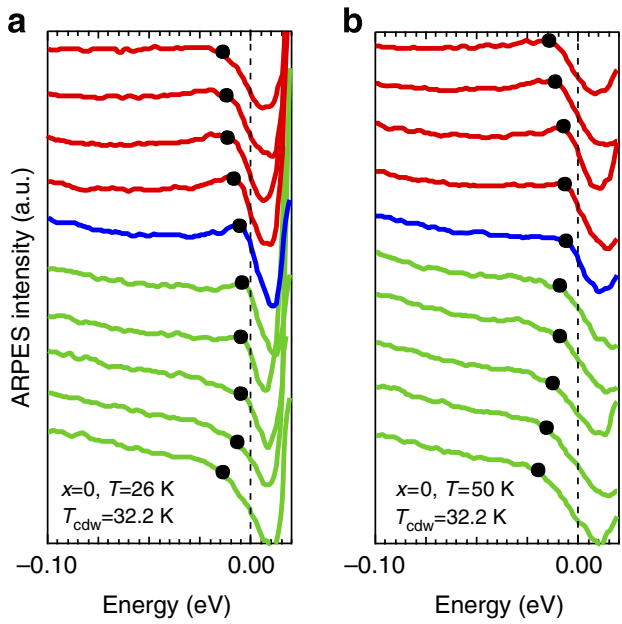

function of temperature, but its width (Fig. 1d) is not resolution limited even at our lowest measured temperature. This suggests that the process of intercalation gives rise to disorder that has impacts on the CDW state, consistent with previous work on $2 \mathrm{H}-\mathrm{NbSe}_{2}$ and other dichalcogenide systems ${ }^{19-22}$. We can nevertheless still identify a CDW transition temperature, even in the intercalated, disordered samples, from a linear extrapolation of the temperature dependence of the superstructure intensity, as indicated in Fig. 1a.

From our XRD data on samples with different concentrations $x$ of intercalant ions, we observe that $T_{\mathrm{cdw}}$ is quickly suppressed (Fig. 1a), as $x$ is increased and beyond $x_{\mathrm{c}}$ the superstructure peaks become very broad, indicating that the CDW order becomes of short range. Qualitatively, similar attributes have been identified in resistivity measurements as well: the $\mathrm{CDW}$-induced anomaly in the resistivity becomes weaker with increasing $x$, shifts to lower temperatures and eventually disappears beyond $x_{\mathrm{c}}$ (see ref. 22, Supplementary Note 1 and Supplementary Fig. 2). We note that the effect of doping on the CDW is the same irrespective of whether the intercalating ion is Mn or Co as observed by STM, discussed later. The disappearance of $T_{\mathrm{cdw}}$ naturally points to the existence of a QPT at $x_{\mathrm{c}}$. Although our measurements show clear signatures of the change in the CDW order across $x_{c}$, as expected at a QPT, we have not been able to characterize this QPT in greater detail due to the difficulty in synthesizing samples with dense-enough $x$-values around $x_{\mathrm{c}}$.

Angle-resolved photoemission. To elucidate the evolution of the electronic structure across $T_{\mathrm{cdw}}$ and $x_{\mathrm{c}}$, we now focus on the $T$ - and $x$-dependent ARPES measurements. We have taken data over a large region of momentum space in the first Brillouin zone (Supplementary Note 2 and Supplementary Fig. 3a). However, we will predominantly be concerned with ARPES data as a function of energy at specific momentum values close to the highsymmetry $K-M$ line (Fig. 2d). The reason for choosing this particular direction is that the CDW gap is maximum along it and hence easily detectable by ARPES 23,24 . The Fermi function (FF) at corresponding temperatures is divided out to better

C

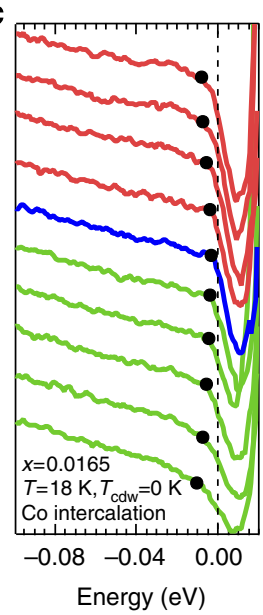

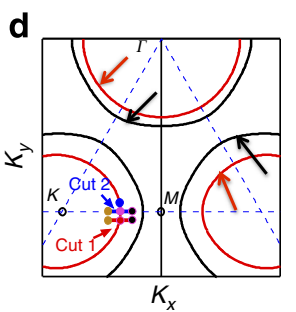

Figure 2 | Electronic dispersion exhibiting back bending. (a,b) FF divided EDCs as a function of momentum $\mathbf{k}$ along cut 1 (defined in d), which is close to the $K-M$ line, for $x=0$, and at $T=26 \mathrm{~K}$ and $T=50 \mathrm{~K}$, respectively. Red EDCs are associated with momenta $\mathbf{k}$ inside the inner Fermi surface (centred around the $K$ point) starting from the brown dot (shown in $\mathbf{d}$ ) to the red dot along cut 1 , green ones to the remaining $\mathbf{k}$ points along cut 1 between the red and black dots. (c) FF divided EDCs for $x=0.0165$ (Co intercalation) at $T=18 \mathrm{~K}$, along cut 2, which is along $K-M$, as shown in d. Red EDCs correspond to $\mathbf{k}$ starting from the brown dot inside the inner Fermi surface (centred around the $K$ point) to the pink dot along cut 2 , green ones to the remaining $\mathbf{k}$ points between the pink and black dots. Blue curves indicate EDCs at the Fermi momentum, the black dots show the locations of the peak and kinks of the EDCs and the black dashed lines are at the chemical potential. The method for locating the black dots is described in Supplementary Note 5 and Supplementary Fig. 7. (d) Fermi surface plot based on the tight-binding fit to the band structure of $2 \mathrm{H}-\mathrm{NbSe}_{2}$ in ref. 24 . Black and red arrows denote outer and inner Fermi surface barrels. 
visualize the existence of the gap $\Delta$ from such energy distribution curves $(\mathrm{EDCs})^{25,26}$. In Fig. 1b, we display the spectral function obtained from the FF-divided EDCs as a function of $T$ for $x=0$ (raw data, shown in the Supplementary Fig. 3b,c, agree with previous ARPES studies $\left.{ }^{23,24}\right)$. At the lowest temperature $\left(T=26 \mathrm{~K}<T_{\text {cdw }}=33 \mathrm{~K}\right)$, the spectrum exhibits a coherence peak at an energy below the chemical potential $\mu$, signalling a non-zero value of the gap. Unlike superconducting or one-dimensional CDW systems, we find that this gap is centred at positive energies, that is, above $\mu$. This particle-hole asymmetry is clearly evident from the FF-divided ARPES data shown in Fig. 1b, which has a negative slope at $\mu$. Consequently, the estimation of the energy gap based on the leading edge or by symmetrizing the spectra around $\mu^{23,24}$ underestimates the size of the gap. Given that the minimum of the FF-divided ARPES intensity occurs above $\mu$, we can not determine the exact magnitude of the energy gap, but can only establish its existence and that, in agreement with recent STM data ${ }^{27}$, it is larger than estimated by symmetrization and leading-edge analysis in previous ARPES studies. The presence of a particle-hole asymmetric gap is an indication that Fermi surface nesting is not important for driving the $\mathrm{CDW}$ in $2 \mathrm{H}-\mathrm{NbSe}_{2}$. This is supported by recent measurements of soft phonon modes using inelastic X-ray scattering ${ }^{16}$. However, differing conclusions about the role of nesting have been offered in previous ARPES studies ${ }^{28-30}$.

With increase in temperature, the intensity of the coherence peak is reduced, but its energy remains the same. Finally, above $T_{\mathrm{cdw}}$, this peak disappears and remarkably, the spectra become almost $T$ independent. Although the spectra for $T>T_{\mathrm{cdw}}$ do not have a discernable peak, they do have a well-defined 'kink', that is, a sharp change of slope. Closer inspection of Fig. $1 \mathrm{~b}$ reveals that the location of this kink in the spectra above $T_{\mathrm{cdw}}$ is similar to that of the coherence peaks below $T_{\mathrm{cdw}}$, as shown by the red dotted line. Although we cannot obtain the exact magnitude of the energy gap as a function of $T$ through $T_{\mathrm{cdw}}$, the fact that peak/ kink structure of the ARPES spectra lies below the chemical potential clearly demonstrates that the energy gap persists for $T>T_{\text {cdw. }}$. On the other hand, there is a loss of phase coherence as indicated by the absence of a peak in these spectra. Our ARPES data on $2 \mathrm{H}-\mathrm{TaS}_{2}$ exhibits similar features (Supplementary Note 3 and Supplementary Fig. 5), which in turn suggests that these features are generic to quasi two-dimensional (2D) CDW systems. We have also seen similar results for other values of $x$ as well in $2 \mathrm{H}-\mathrm{NbSe}_{2}$ (Supplementary Fig. 4). Therefore, $T_{\text {cdw }}$ can be identified as the transition from an incoherent, gapped electronic state to a coherent one. This implies that although the CDW order parameter vanishes above $T_{\mathrm{cdw}}$, its amplitude does not.

In Fig. 1e, we display the normalized spectral weight as a function of $T$ (the details of the normalization procedure is described in the caption of Fig. 1). One can clearly observe that it decays monotonically with $T$ and vanishes above $T_{\mathrm{cdw}}$. The $x$ dependence of the spectra (Fig. 1c) through $x_{\mathrm{c}}$ resembles the $T$ dependence through $T_{\mathrm{cdw}}$, for example, the spectrum at the lowest measured temperature progressively loses its peak with increasing $x$ and eventually becomes featureless for $x>x_{c}$. It can readily be seen (Fig. 1f) that the $x$ dependence of the normalized coherent spectral weight follows a similar trend as the $T$ dependence, that is it steadily goes down with increasing $x$ and vanishes above $x_{\mathrm{c}}$. However, a finite energy gap persists for all $x$, even beyond $x_{\mathrm{c}}$. In other words, there exists an extended region in the $(x, T)$ phase plane of $2 \mathrm{H}-\mathrm{NbSe}_{2}$ systems where the spectral function has an energy gap but no coherence.

We now look at the details of the $(x, T)$ phase plane in the context of pairing. As a direct consequence of particle-hole coupling, the electronic dispersion, that is, the relation between electronic energy and momentum, is altered from the one in the normal state. In the CDW state with ordering wave vector $\mathbf{q}$, an electronic state with momentum $\mathbf{k}$ gets coupled to another with momentum $\mathbf{k}+\mathbf{q}$, and the dispersion is modified to $E_{\mathbf{k}}=1 / 2\left(\varepsilon_{\mathbf{k}}+\right.$ $\left.\varepsilon_{\mathbf{k}+\mathbf{q}}\right) \pm\left[\frac{1}{4}\left(\varepsilon_{\mathbf{k}}-\varepsilon_{\mathbf{k}+\mathbf{q}}\right)^{2}+\Delta_{\mathbf{k}}^{2}\right]^{1 / 2}$, where $\Delta_{\mathbf{k}}$ is the energy gap and $\varepsilon_{\mathbf{k}}$ is the normal-state dispersion ${ }^{1,6}$. The dispersion of the lower branch deviates from $\varepsilon_{\mathbf{k}}$, as $\varepsilon_{\mathbf{k}}$ approaches $\varepsilon_{\mathbf{k}+\mathbf{q}}$, reaches a maximum when $\varepsilon_{\mathbf{k}}=\varepsilon_{\mathbf{k}+\mathbf{q}}$ and then bends back to follow $\varepsilon_{\mathbf{k}+\mathbf{q}}$ instead (Supplementary Fig. 6 and Supplementary Note 4). This bending back effect, commonly known as the Bogoliubov dispersion $^{2}$, occurs in the superconducting state due to electron-electron pairing and has been directly observed by ARPES in cuprates ${ }^{31-33}$. As shown in Fig. 2a, by monitoring the peak positions and kinks of the EDCs (see Supplementary Note 5 and Supplementary Fig. 7 for details), it is easy to see that the electronic dispersion below $T_{\mathrm{cdw}}$ for $x=0$ has the expected bending back behaviour. In addition, quite remarkably, the bending back of the dispersion is visible even when the CDW is short ranged-(i) in the $x=0$ sample at $T \sim 50 \mathrm{~K}>T_{\text {cdw }}$ in Fig. $2 \mathrm{~b}$ and (ii) an intercalated sample with $x \sim 0.0165>x_{\mathrm{c}}$ at $T=18 \mathrm{~K}$ in Fig. 2c. The presence of a CDW gap below and above $T_{\mathrm{cdw}}$ along with the persistence of a back bending feature in the electronic dispersion naturally suggests that the electron-hole pairs induced by the CDW persist above $T_{\mathrm{cdw}}$ and beyond $x_{\mathrm{c}}$. We point out that similar observations have been made in the pseudogap phase of cuprates where, just as in the superconducting state, a Bogoliubov dispersion was found to exist above $T_{\mathcal{c}}$, which was considered as evidence for pairing without phase coherence ${ }^{34}$. Taking into consideration of all these observations, one can realize that for $T>T_{\mathrm{cdw}}$ or $x>x_{\mathrm{c}}$ in the $(x, T)$ phase plane of $2 \mathrm{H}-\mathrm{NbSe}_{2}$, there are electron-hole pairs without coherence, which in turn implies that $\mathrm{CDW}$ order parameter vanishes because of phase incoherence.

Scanning tunnelling microscopy. So far, we have concentrated on the structure and the relevant electronic excitations associated with the CDW order in momentum space. To visualize them in real space, we have performed high-resolution STM measurements as a function of $T$ and $x$ as shown in Fig. 3, where the upper panels are STM topographic images, each of which covers an area of $18.5 \mathrm{~nm}$ by $18.5 \mathrm{~nm}$, the second row shows Fourierfiltered images and the bottom panels are 2D-Fourier transform (FT) images. Figure $3 \mathrm{a}$ corresponds to the $x=0$ sample at $T=4.2 \mathrm{~K}$. The almost perfect hexagonal lattice pattern confirms that our pristine samples are of very high quality and practically defect free. A close inspection of the topographic image clearly reveals the presence of a locally commensurate CDW pattern that repeats after every third lattice spacing; however, phase slips lead to the slight incommensuration observed in XRD as well as in the FT of the STM image shown in Fig. $3 \mathrm{i}^{35}$. The long-range order of both the lattice as well as the CDW is reflected in the sharp peaks observed in the FT image, where the outermost set of the six strong peaks correspond to the Bragg peaks of the underlying lattice, and the innermost ones at $\sim 1 / 3$ the wave vector correspond to the lowest-order CDW superlattice peaks (Figs 3 and 4). Our topographic as well as FT images of pure samples are consistent with earlier STM measurements ${ }^{27,36-38}$. Although the $\mathrm{CDW}$ order is well visible in the topographic image of the pure sample, the CDW corrugation in $\mathrm{NbSe}_{2}$ is very small, and to enhance the subtle features due to CDW modulations we show Fourier-filtered images in the middle row, panels e-h of Fig. 3. These images are obtained by applying a mask to the FT image that only retains the region around the lowest-order CDW peaks, for example, the region between the dash-dotted circles shown in Fig. 3i, and transforming back to real space. This results in images of the CDW maxima and allows a direct visualization of the phase 

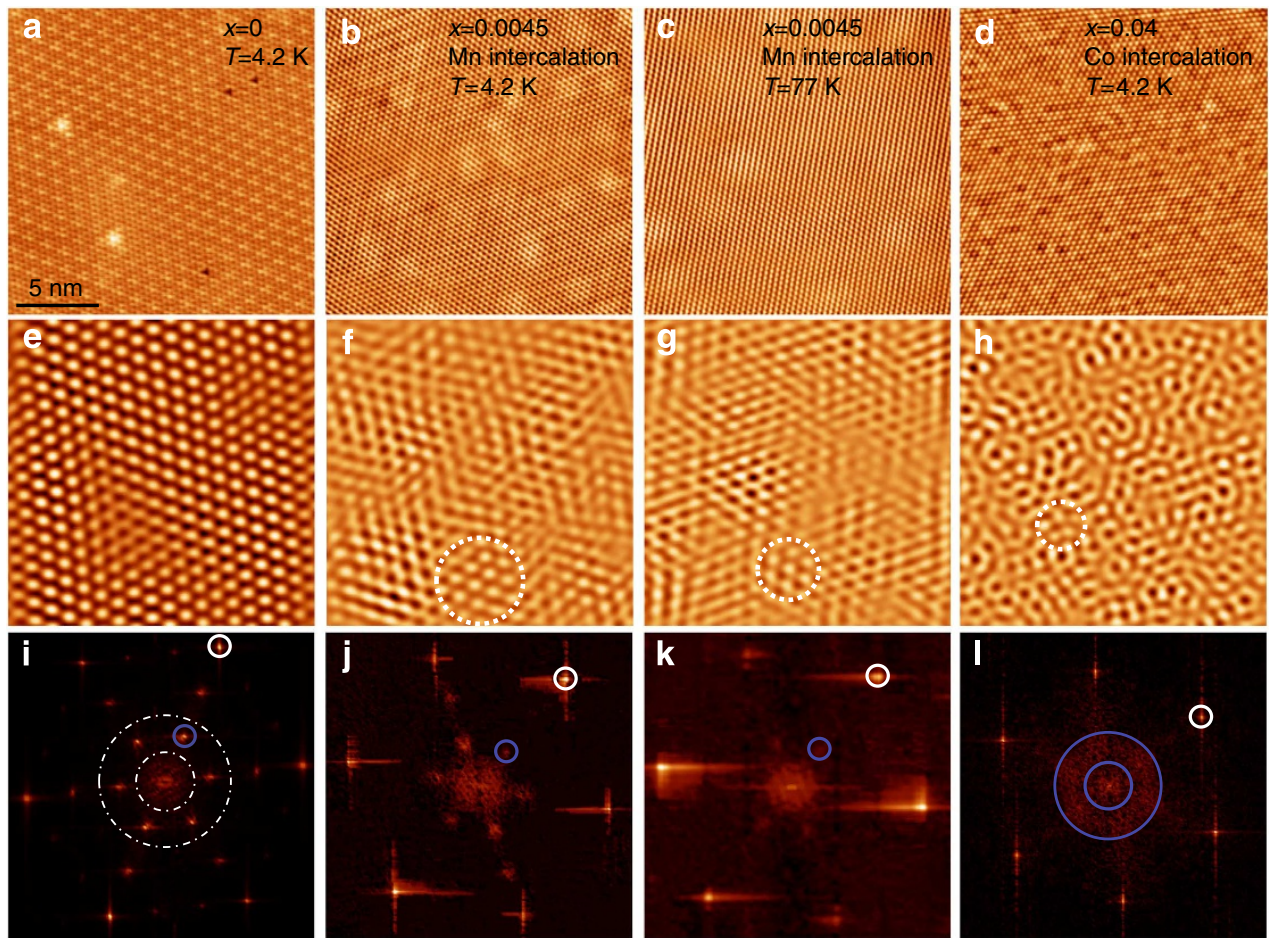

Figure 3 | STM data on pristine and intercalated $\mathbf{2} \mathbf{H}-\mathbf{N b S e}_{\mathbf{2}}$ samples. STM topographic images of $(\mathbf{a}) x=0$ at $4.2 \mathrm{~K},(\mathbf{b}, \mathbf{c}) x=0.0045(\mathrm{Mn}$ intercalated) at 4.2 and $77 \mathrm{~K}$, respectively, and (d) $x=0.04$ (Co intercalated) at $4.2 \mathrm{~K}$. The scan areas are $18.5 \mathrm{~nm} \times 18.5 \mathrm{~nm}$. The images have been acquired at $V=+50 \mathrm{mV}$ and $I=100 \mathrm{pA}$. The total $z$-deflection is $0.6 \AA$ in $\mathbf{a}, \mathbf{c}, 1.0 \AA$ in $\mathbf{b}$ and $1.2 \AA$ in $\mathbf{d}$. (e-h) Fourier-filtered images obtained by retaining only the region around the lowest-order CDW peak in the FT images, for example, the region $0.2 q_{\text {BRAGG }}<q<0.45 q_{\text {BRAGG }}$ indicated by the white dash-dotted circles in $\mathbf{i}$, and transforming back to real space. The dashed white circles in these filtered images show examples of patches with well-defined CDW structures in the intercalated samples. (i-I) 2D-FTs of the topographic images in a-d. The white circles indicate the position of one of the lowest-order Bragg peaks at $q_{\text {BRAGG; }}$ blue circles in $\mathbf{i}-\mathbf{k}$ at $\sim 1 / 3 q_{\text {Bragg }}$ indicate the position of the lowest-order CDW superlattice peaks. In samples with high CDW disorder, the FT does not show clear CDW peaks but rather a ring-like structure, as indicated by the blue circles in $\mathbf{I}$ for $\mathrm{Co}_{0.04} \mathrm{NbSe}_{2}$.
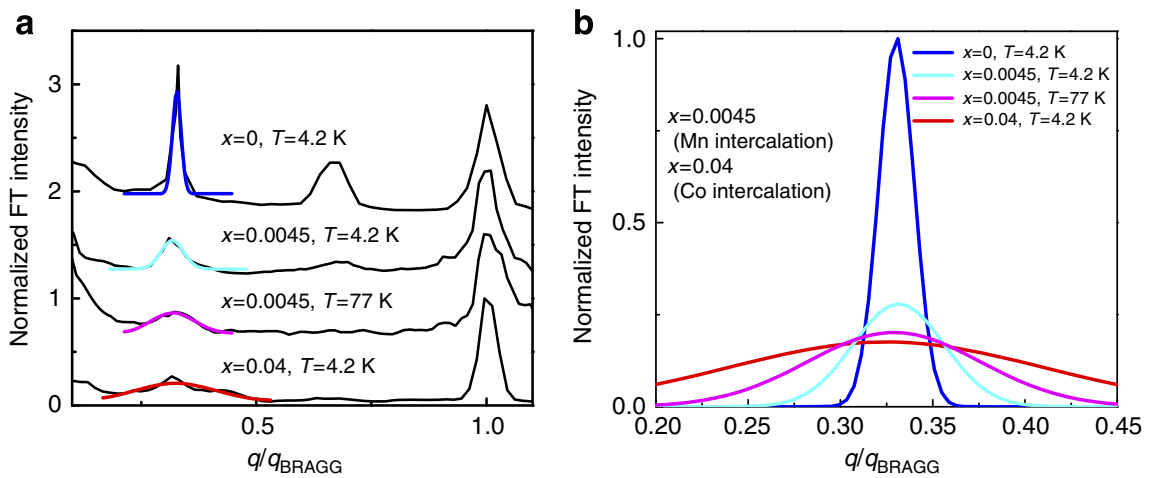

Figure 4 | STM FT profiles. (a) Line profiles of the FT images in Fig. 3i-I along one of the lattice wave vector directions. The curves have been shifted for clarity. The intensity of each profile has been normalized to the intensity of the Bragg peak. The CDW peak at $q=q_{\text {BRAGG }} / 3$ has been fitted with a Gaussian and the fit is superimposed on the FT line profile for each curve. (b) Gaussian fits of the CDW peaks in a normalized to the CDW peak of pure $\mathrm{NbSe}_{2}$.

and CDW domain structure. To ensure that the filtered images are free from possible artefacts due to the filtering procedure, we compared images obtained using different sizes of the filtering mask. The main features of the filtered images remain the same regardless of the filter used. The long-range order and phase coherence of the CDW in the pure sample is clearly reflected in its Fourier-filtered image, where an almost perfect CDW lattice is obtained (Fig. 3e). STM topographic images of intercalated samples reveal the presence of defects, both at the surface and beneath the surface, as bright spots. In the sample with nominal doping $x=0.0045$ (Fig. 3b), the local density of defects in the topographic image is in the range $0.006<x<0.0075$. In contrast to the pure sample, the Fourier-filtered image (Fig. $3 \mathrm{f}$ ) reveals that coherent CDW order does not exist uniformly over the entire sample, but only in the form of patches. As a demonstration, we have indicated one such region with locally well-defined CDW order in the Fourier-filtered image (Fig. 3f) by a dashed circle. In other words, CDW order in the intercalated sample with $x=0.0045$ lacks long-range translational order, which in turn supports the observation, as in Fig. 1, that the CDW order in this intercalated sample is not long range. Consistent with this, the CDW peaks of the FT image in Fig. $3 j$ are broader than those of 


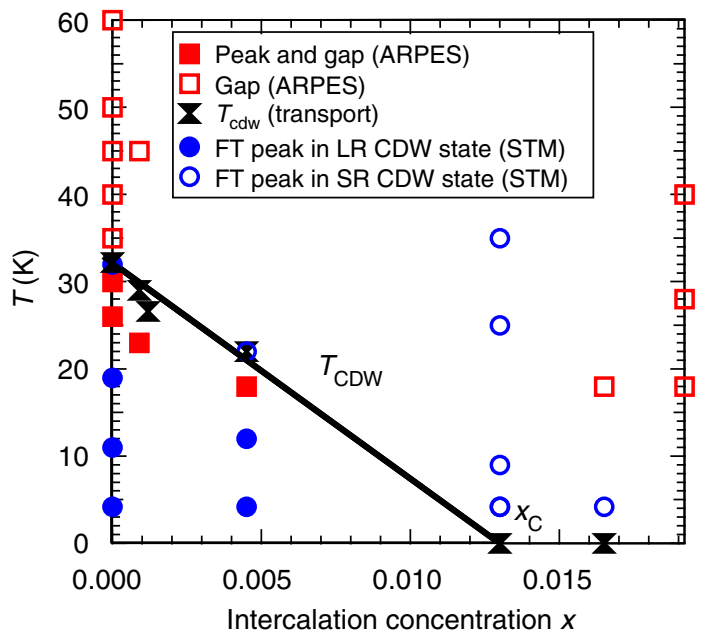

Figure 5 | Intercalation versus temperature diagram of CDW correlations in $\mathbf{2 H}-\mathbf{N b S e}_{2}$. Overview plot of the results from ARPES, STM, transport and XRD measurements. ARPES data points are shown by red squares. Filled squares correspond to the simultaneous presence of the coherence peak and an energy gap, empty ones to the presence of only an energy gap. STM data points are shown by blue circles, indicating the presence of a CDW. Bow ties denote $T_{\mathrm{cdw}}$, obtained from transport measurements, marking the transition from a long-range-ordered CDW state to a short-range-ordered state as a function of $x$ and $T$. Extrapolation of this phase line leads to $x_{c} \sim 0.013$, beyond which there is only short-range order at all measured temperatures. The superconducting transitions, observed for all samples except the very highest doping as listed in Supplementary Table 1, are not shown in this diagram.

the pure sample (Figs $3 \mathrm{i}$ and 4). As $x$ is increased to 0.04 , the size of the patches with coherent CDW order (Fig. 3d,h) is further reduced and the peaks in the FT become even broader, leading to a ring-like structure (Fig. 31). In Fig. 4a, we present the line profiles of the FT in Fig. 3i-l along the atomic wave vectors as a function of doping, and in Fig. $4 \mathrm{~b}$ we display the Gaussian fits to these CDW peaks (Supplementary Note 6), from which we clearly see the broadening of the CDW peak with doping. These results simply relate to the fact that the CDW coherence length decays with increasing $x$ and eventually becomes short range, consistent with our ARPES and XRD observations. We note that these observations are independent of the intercalating ion, see Supplementary Note 7 and Supplementary Figs 8 and 9. STM observations of domain-like structures having well-defined CDW order, similar to those shown in Fig. 3f, have been reported in a number of disordered dichalcogenide systems ${ }^{39,40}$.

We now focus on STM data for $T>T_{\text {cdw }}$ (Fig. 3c,g,k) corresponding to the $x=0.0045$ sample at $T=77 \mathrm{~K}>T_{\text {cdw }}=20$ $\mathrm{K}$. Although weak in intensity, well-defined CDW modulations survive in various regions, as best seen in the Fourier-filtered image Fig. 3g, and the FT image (Fig. 3k) still clearly shows broad $\mathrm{CDW}$ peaks, indicating the persistence of short-range CDW correlations to temperatures well above $T_{\mathrm{cdw}}$. In the pure sample, we observe very weak CDW modulations localized around the impurities up to $45 \mathrm{~K}$ (Supplementary Fig. 10a). Given the small amount of defects and the weak modulation, only very weak CDW peaks are observed in the 2D-FT images. We do not observe any sign of CDW modulations in real space or in 2D-FT at $77 \mathrm{~K}$ (Supplementary Fig. 10b). This can be understood by realizing that the STM images represent a time-averaged view of the sample, and that in regions far from any pinning defects the signatures of short-range order are averaged out by dynamic fluctuations.

\section{Discussion}

The phase diagram of the CDW correlations in intercalated $2 \mathrm{H}-\mathrm{NbSe}_{2}$ shown in Fig. 5 summarizes our results (for simplicity, the superconducting transition observed in all but the very highest doped sample are not shown in this figure). The squares are based on the ARPES spectra that are: (1) coherent and gapped (solid squares) within the triangular region where the CDW order is present; and (2) incoherent and gapped (empty squares) outside of this triangle, indicating short-range CDW order instead. The circles correspond to FT-STM data-solid ones within the triangular region where long-range CDW order exist and empty ones outside where CDW order is of short range. The bow ties indicate the CDW transition temperature determined from transport measurements and are included for completeness.

This phase diagram closely resembles that of the transition from the superconducting to the pseudogap state in cuprates ${ }^{41}$, and that of the superconducting to insulating transition in disordered systems ${ }^{9,13,42}$. The experimental observation of the persistence of an energy gap and the disappearance of single-particle coherence across classical or QPTs seems to be a common feature of these entirely different systems. The enigmatic pseudogap phase of cuprates is in many ways the most complicated of these examples, with close proximity to a Mott insulator and various competing orders. In this respect, the simplicity of $2 \mathrm{H}-\mathrm{NbSe}_{2}$ studied in this paper makes it possible to focus on a single-order parameter, associated with CDW formation, and examine in detail how the spectroscopic signatures evolve across the QPT where this ordering is destroyed.

\section{Methods}

Intercalated $\mathbf{2} \mathbf{H}-\mathbf{N b S e}_{2}$ samples. Cobalt (Co) and manganese (Mn) have been intercalated in pristine $2 \mathrm{H}$-NbSe $\mathrm{Nb}_{2}$ single crystals by adopting the procedures explained in refs 43,44 . XRD patterns show that our intercalated samples have the same structure as the pristine $2 \mathrm{H}-\mathrm{NbSe}_{2}$ samples. The concentration of Co and $\mathrm{Mn}$ were determined from energy-dispersive X-ray microprobe analysis measurements. While performing energy-dispersive X-ray microprobe analysis on intercalated samples, we have randomly chosen different regions of the samples and found that the atomic concentrations of $\mathrm{Co}$ and $\mathrm{Mn}$ remain almost the same, meaning there is no evidence for clustering on the length scale of $100 \mathrm{~nm}$. This is substantiated by previous extended $\mathrm{x}$-ray absorption fine structure (EXAFS) measurements ${ }^{45}$.

The superconducting critical temperature $\left(T_{\mathrm{c}}\right)$ for pristine $2 \mathrm{H}-\mathrm{NbSe}_{2}$ samples is $\sim 7.2 \mathrm{~K}$ and it was previously shown that $T_{\mathrm{c}}$ gets suppressed due to intercalation ${ }^{46}$ Our magnetization versus temperature measurements for different samples show sharp superconducting transitions (Supplementary Fig. 1), which further support that our intercalated samples are homogeneous. Supplementary Table 1 lists details of all the samples used in this study.

Transport measurements. The temperature-dependent resistivity of the single crystals was measured using regular four-terminal transport measurement technique performed with a Physical Property Measurement System by Quantum Design equipped with external device control option. Keithley 6220 current source and Keithley 2182 nanovoltmeter were used to apply a DC current and measure the voltage drop, respectively.

XRD measurements. Synchrotron XRD experiments were performed at the Advanced Photon Source, Argonne National Laboratory. Data were taken in transmission geometry with $22.3 \mathrm{keV} \mathrm{X}$-rays on the 11 -ID-D beamline, using a Ketek Silicon Drift point detector, and on the high-energy station of beamline 6-ID-D, with $80 \mathrm{keV} \mathrm{X}$-rays and a Pilatus $100 \mathrm{~K}$ area detector. In both setups, the samples were mounted on the cold head of a closed-cycle helium displex refrigerator and sealed inside a Be-can with He exchange gas.

ARPES measurements. ARPES measurements were carried out at the PGM beamline of the Synchrotron Radiation Center, Stoughton, WI, and at the SIS beamline of the Swiss Light Source, Paul Scherrer Institut, Switzerland, using Scienta R4000 analysers and $22 \mathrm{eV}$ photons for all the measurements. Typical spot sizes of the synchrotron beam on the sample's surface is $\sim 50-150 \mu \mathrm{m}$ times $300 \mu \mathrm{m}$.

STM measurements. Low-temperature STM measurements have been performed using a Unisoku UHV STM system (Unisoku USM-1300 combined with RHK Electronics), with a base pressure of $1.0 \times 10^{-10}$ Torr. The samples were cleaved 
just before cooling down. Atomically resolved images were acquired in the constant current mode with a constant voltage between sample and tip. We used Pt-Ir tips in all of our experiments.

\section{References}

1. Gruner, G. Density Waves in Solids (Perseus Publishing, Cambridge, 1994).

2. Schrieffer, J. R. Theory of Superconductivity (Benjamin, New York, 1964).

3. Emery, V. J. \& Kivelson, S. A. Importance of phase fluctuations in superconductors with small superfluid density. Nature 374, 434-437 (1995).

4. McMillan, W. L. Microscopic model of charge-density waves in $2 \mathrm{H}-\mathrm{TaSe}_{2}$. Phys. Rev. B. 16, 643-650 (1977).

5. Varma, C. M. \& Simons, A. L. Strong-coupling theory of charge-density-wave transitions. Phys. Rev. Lett. 51, 138-141 (1983).

6. Rossnagel, K. On the origin of charge-density waves in select layered transitionmetal dichalcogenides. J. Phys. Condens. Matter 23, 213001 (2011).

7. Taraphder, A., Koley, S., Vidhyadhiraja, N. S. \& Laad, M. S. Preformed excitonic liquid route to a charge density wave in $2 \mathrm{H}-\mathrm{TaSe}_{2}$. Phys. Rev. Lett. 106, 236405 (2011)

8. Kuchinskii, E. Z. et al. Electronic structure of two-dimensional hexagonal diselenides: charge density waves and pseudogap behavior. JETP 114, 671-680 (2012).

9. Bouadim, K. et al. Single- and two-particle energy gaps across the disorderdriven superconductor-insulator transition. Nat. Phys. 7, 884-889 (2011).

10. Ding, H. et al. Spectroscopic evidence for a pseudogap in the normal state of underdoped high- $\mathrm{T}_{\mathrm{c}}$ superconductors. Nature 382, 51-54 (1996).

11. Loeser, A. G. et al. Excitation gap in the normal state of underdoped $\mathrm{Bi}_{2} \mathrm{Sr}_{2} \mathrm{CaCu}_{2} \mathrm{O}_{8+\delta}$. Science 273, 325-329 (1996).

12. Xu, Z. A. et al. Vortex like excitations and the onset of superconducting phase fluctuation in underdoped $\mathrm{La}_{2}{ }_{x} \mathrm{Sr}_{x} \mathrm{CuO}_{4}$. Nature 406, 486-488 (2000).

13. Sacepe, B. et al. Localization of preformed Cooper pairs in disordered superconductors. Nat. Phys. 7, 239-244 (2011).

14. Feld, M. et al. Observation of a pairing pseudogap in a two-dimensional Fermi gas. Nature 480, 75-78 (2011).

15. Valla, T. et al. Quasiparticle spectra, charge-density waves, superconductivity, and electron-phonon coupling in $2 \mathrm{H}-\mathrm{NbSe}_{2}$. Phys. Rev. Lett. 92, 086401 (2004).

16. Weber, F. et al. Extended phonon collapse and the origin of the charge-density wave in $2 \mathrm{H}-\mathrm{NbSe}_{2}$. Phys. Rev. Lett. 107, 107403 (2011).

17. Moncton, D. E., Axe, J. D. \& DiSalvo, F. J. Study of superlattice formation in $2 \mathrm{H}-\mathrm{NbSe}_{2}$ and $2 \mathrm{H}-\mathrm{TaSe}_{2}$ by neutron scattering. Phys. Rev. Lett. 34, 734-737 (1975).

18. Malliakas, C. D. \& Kanatzidis, M. G. Nb-Nb interactions define the charge density wave structure of $2 \mathrm{H}-\mathrm{NbSe}_{2}$. J. Am. Chem. Soc. 135, 1719 (2013).

19. Mutka, H. et al. Charge-density waves and localization in electron-irradiated 1T-TaS 2 . Phys. Rev. B. 23, 5030-5037 (1981).

20. Tao, W. H. et al. Effect of Te doping on superconductivity and charge-density wave in dichalcogenides $2 \mathrm{H}-\mathrm{NbSe}_{2}{ }_{x} \mathrm{Te}_{x}(x=0,0.1,0.2)$. Chinese Phys. 16, 2471-2474 (2007)

21. Mutka, H. Superconductivity in irradiated charge-density-wave compounds $2 \mathrm{H}-\mathrm{NbSe}_{2}, 2 \mathrm{H}-\mathrm{TaS}_{2}$, and $2 \mathrm{H}-\mathrm{TaSe}_{2}$. Phys. Rev. B 28, 2855-2858 (1983).

22. Iavarone, M. et al. STM studies of $\mathrm{Co}_{x} \mathrm{NbSe}_{2}$ and $\mathrm{Mn}_{x} \mathrm{NbSe}_{2}$. J. Phys: Conf. Ser. 150, 052073 (2009).

23. Borisenko, S. V. et al. Two energy gaps and Fermi-surface "Arcs" in $\mathrm{NbSe}_{2}$. Phys. Rev. Lett. 102, 166402 (2009).

24. Rahn, D. J. et al. Gaps and kinks in the electronic structure of the superconductor $2 \mathrm{H}-\mathrm{NbSe}_{2}$ from angle-resolved photoemission at $1 \mathrm{~K}$. Phys. Rev. B 85, 224532 (2012).

25. Damascelli, A., Hussain, Z. \& Shen, Z. X. Angle resolved photoemission studies of the cuprate superconductors. Rev. Mod. Phys. 75, 473-541 (2003).

26. Campuzano, J. C., Norman, M. R. \& Randeria, M. in Physics of Superconductors vol. II (eds Bennemann, K. H. \& Ketterson, J. B.) 167-273 (Springer, Berlin, 2004).

27. Soumyanarayanan, A. et al. Quantum phase transition from triangular to stripe charge order in $\mathrm{NbSe}_{2}$. Proc. Natl Acad. Sci. 110, 1623-1627 (2013).

28. Tonjes, W. C. et al. Charge-density-wave mechanism in the $2 \mathrm{H}-\mathrm{NbSe}_{2}$ family: angle-resolved photoemission studies. Phys. Rev. B 63, 235101 (2001).

29. Straub, T. h. et al. Charge-density-wave mechanism in $2 \mathrm{H}-\mathrm{NbSe}_{2}$ : photoemission results. Phys. Rev. Lett. 82, 4504-4507 (1999).

30. Shen, D. W. et al. Primary role of the barely occupied states in the charge density wave formation of $\mathrm{NbSe}_{2}$. Phys. Rev. Lett. 101, 226406 (2008).

31. Matsui, H. et al. BCS-like Bogoliubov quasiparticles in high- $T_{\mathrm{c}}$ superconductors observed by angle resolved photoemission spectroscopy. Phys. Rev. Lett. 90, 217002 (2003)

32. Campuzano, J. C. et al. Direct observation of particle-hole mixing in the superconducting state by angle-resolved photoemission. Phys. Rev. B 53, R14737-R14740 (1996).

33. Hashimoto, M. et al. Particle-hole symmetry breaking in the pseudogap phase of Bi2201. Nat. Phys. 6, 414-418 (2010).

34. Kanigel, A. et al. Evidence for pairing above the transition temperature of cuprate superconductors from the electronic dispersion in the pseudogap phase. Phys. Rev. Lett. 101, 137002 (2008).
35. McMillan, W. L. Theory of discommensurations and the commensurateincommensurate charge-density-wave transition. Phys. Rev. B 14, 1496-1502 (1976).

36. Giambattista, B. et al. Charge-density waves observed at $4.2 \mathrm{~K}$ by scanningtunneling microscopy. Phys. Rev. B. 37, 2741-2744 (1988).

37. Mallet, P. et al. Spatial and energy variation of the local density of states in the charge density wave phase of $2 \mathrm{H}-\mathrm{NbSe}_{2}$. J. Vac. Sci. Technol. B 14, 1070-1074 (1996).

38. Arguello, C. J. et al. Visualizing the charge density wave transition in $2 \mathrm{H}-\mathrm{NbSe}_{2}$ in real space. Phys. Rev. B 89, 235115 (2014).

39. Dai, H. et al. Weak pinning and hexatic order in a doped two-dimensional charge-density-wave system. Phys. Rev. Lett. 66, 3183-3187 (1991).

40. Iwaya, K. et al. Electronic state of $\mathrm{NbSe}_{2}$ investigated by STM/STS. Phys. B 329-333, 1598-1599 (2003).

41. Chatterjee, U. et al. Electronic phase diagram of high-temperature copper oxide superconductors. Proc. Natl Acad. Sci. 108, 9346-9349 (2011).

42. Baturina, T. I. et al. Localized superconductivity in the quantum-critical region of the disorder-driven superconductor-insulator transition in TiN thin films. Phys. Rev. Lett. 99, 257003 (2007).

43. Beal, A. R. in Intercalated Layer Materials (ed. Lévy, F.) 251 (Reidel D., Dordrecht, 1979).

44. Oglesby, C. S. et al. Growth of faceted niobium diselenide. J. Cryst. Growth 137, 289-294 (1994).

45. Weber, F. et al. Extended X-ray absorption fine structure spectroscopy in $\mathrm{Co}_{0.013} \mathrm{NbSe}_{2}$. J. Phys. Conf. Ser. 200, 012224 (2010).

46. Iavarone, M. et al. Effect of magnetic impurities on the vortex lattice properties in $\mathrm{NbSe}_{2}$ single crystals. Phys. Rev. B 78, 174518 (2008).

\section{Acknowledgements}

Work at Argonne (U.C., J.Z., J.P.C., C.D.M., M.G.K., H.C., J.P.C.R., F.W., J.C.C, R.O., M.R.N., S.R.) was supported by the Materials Science and Engineering Division, Basic Energy Sciences, Office of Science, U.S. Department of Energy. Work at Temple University (M.I) and Drexel University (G.K.) was supported as part of the Center for the Computational Design of Functional Layered Materials, an Energy Frontier Research Center funded by the U.S. DOE, BES under Award DE-SC0012575. J.v.W. acknowledges support from a VIDI grant financed by the Netherlands Organization for Scientific Research (NWO). M.R. was supported by the DOE-BES grant DE-SC0005035. N.T. was supported by the U.S. DOE, Office of Science, Grant DE-FG02-07ER46423. The Synchrotron Radiation Center is supported by the University of Wisconsin, Madison. Synchrotron X-ray scattering experiments were carried out at the Advanced Photon Source, which is supported by the DOE, Office of Science, BES. We thank Ming Shi for his support with the experiments at the Swiss Light Source, Paul Scherrer Institut, Switzerland, and D. Robinson and K. Attenkofer for their support with the XRD measurements at the Advanced Photon Source, Argonne National Laboratory.

\section{Author contributions}

U.C. and S.R. proposed the research project. U.C. and J.Z. carried out the ARPES measurements and analysis of the ARPES data. J.P.C., J.P.C.R., F.W., R.O. and S.R carried out the XRD measurements and analysis of the XRD data. M.I., R.D.C. and G.K. carried out the STM, transport measurements and analysis of STM and transport data M.I., G.K., C.D.M. and M.G.K. synthesized high-quality pristine and intercalated $\mathrm{NbSe}_{2}$ samples. H.C. conducted the superconducting SQUID measurements on the samples, to determine their superconducting critical temperatures. U.C., J.v.W., M.R., N.T., M.R.N. and S.R. wrote the manuscript. All authors discussed the results and contributed to the manuscript.

\section{Additional information}

Supplementary Information accompanies this paper at http://www.nature.com/ naturecommunications

Competing financial interests: The authors declare no competing financial interests

Reprints and permission information is available online at http://npg.nature.com/ reprintsandpermissions/

How to cite this article: Chatterjee, U. et al. Emergence of coherence in the charge-density wave state of $2 \mathrm{H}-\mathrm{NbSe}_{2}$. Nat. Commun. 6:6313 doi: 10.1038/ncomms7313 (2015).

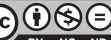

This work is licensed under a Creative Commons AttributionNonCommercial-NoDerivs 4.0 International License. The images or other third party material in this article are included in the article's Creative Commons license, unless indicated otherwise in the credit line; if the material is not included under the Creative Commons license, users will need to obtain permission from the license holder to reproduce the material. To view a copy of this license, visit http:// creativecommons.org/licenses/by-nc-nd/4.0/ 